ISSN 2447-9071

doi https://doi.org/10.36414/rbmc.v6i15.54

Contato para correspondência:

Graziela Torres Blanch

E-mail:

grazielatb@gmail.com

Conflito de interesse: Não

Financiamento: Recursos próprios

Recebido: $10 / 08 / 2020$

Aprovado: $17 / 08 / 2020$

\section{Prevalência de dislipidemia em indivíduos atendidos no laboratório de um hospital de Goiânia - GO}

\section{Prevalence of dyslipidemia in individuals attended in the laboratory of a hospital in Goiânia - GO}

André Guimarães de Paula', Paulo Tadeu Silva Campos', Monatha Nayara Guimarães Teófilo', Clayson Moura Gomes ${ }^{1,2}$, Sérgio Henrique Nascente Costa ${ }^{1,2,3}$, Graziela Torres Blanch ${ }^{1}$

'Pontifícia Universidade Católica de Goiás - PUC Goiás

${ }^{2}$ Faculdade da Polícia Militar - FPM

${ }^{3}$ Universidade Federal de Goiás - UFG

\section{Resumo}

Este estudo teve por objetivo identificar a prevalência de dislipidemias de acordo com sexo e faixa etária de individuos atendidos em um hospital de Goiânia-GO. Trata-se de um estudo do tipo observacional, transversal, realizado a partir de dados disponibilizados, no período de fevereiro/2017 a março/2018. A amostra representativa consistiu de indivíduos com alteração no perfil lipídico independente do gênero e da faixa etária. Os valores referenciais e a classificação das dislipidemias seguiram os critérios da Atualização da Diretriz Brasileira de Dislipidemias e Prevenção da Aterosclerose -2017. Os resultados foram tabuladosem Microsoft Excel ${ }^{\oplus}$, analisados em BioStat ${ }^{\varpi}$, considerando $p<0,05$ (IC95\%). Estudo aprovado pelo Comitê de Ética da Pontifícia Universidade Católica de Goiás. Contou com uma população de 7699 pacientes, destes 3014 (39\%) eram do sexo feminino e 4685 (61\%) do sexo masculino. Cerca de 69\% dos pacientes apresentaram perfil lipídico alterado. Amaior prevalência de paciente com HDL-Cbaixo, foido sexofeminino com 44\%,jáopredomínio da hiperlipidemia mista foidosexomasculinocom 10\%, ambos independente da faixa etária e sendo $p<0,0001$ (IC95\%). Amaioria da população estudada apresentou dislipidemia, sendo evidente a maior prevalência de HDL-C baixo no sexo feminino e de hiperlipidemia mistanosexomasculino. Talobservaçãodemonstra anecessidade deinvestigação de alterações noperfil lipídico para diagnóstico precoce e prevenção de doenças cardiovasculares.

Palavras-Chave: Dislipidemia, Epidemiologia, Colesterol, Fatores de Risco, Doenças Cardiovasculares.

\begin{abstract}
This study aimed to identify the prevalence of dyslipidemia according to sex and age group of individuals seen at a hospital in Goiânia-GO. This is a observational, cross-sectional study, carried out from data made available from February/2017 to March/2018. The representative sample consisted of individuals with altered lipid profiles regardless of gender and age group. The reference values and the classification of dyslipidemias followed the criteria for updating the Brazilian Guidelines on Dyslipidemias and Atherosclerosis Prevention - 2017. The results were tabulated in Microsoft Excel ${ }^{\oplus}$, analyzed in BioeStat ${ }^{\oplus}$, considering $p<0.05(95 \% \mathrm{Cl}$ ). Study approved by the Ethics Committee of the Pontifical Catholic University of Goiás. This study had a population of 7699 patients, of whom 3014 (39\%) were female and 4685 (61\%) male. About 69\% of the patients had an altered lipid profile. The highest prevalence of patients with low HDL-C was female, with 44\%, whereas the predominance of mixed hyperlipidemia was male, with 10\%, both regardless of age group and with $p<0.0001$ (95\% CI). The majority of the studied population had dyslipidemia, with a higher prevalence of low HDL-C in females and mixed hyperlipidemia in males being evident. Such observation demonstrates the need to investigate changes in the lipid profile for early diagnosis and prevention of cardiovascular diseases.
\end{abstract}




\section{Introdução}

As doenças cardiovasculares (DCV) representam uma das principais causas de mortes no cenário mundial, sendo consideradas um grande problema de saúde pública. Dados da Organização Mundial da Saúde (OMS) mostram que, nas últimas décadas, das 50 milhões de mortes ocorridas no mundo, 31\% foram devidas a doenças cardiovasculares, o que representa cerca de 17 milhões de pessoas ${ }^{1-3}$. No Brasil, as DCV são as que apresentam maiores gastos entre as causas de internações hospitalares no país4. Estima-se que a despesa com medicações representem $10 \%$ do custo total da assistência farmacêutica popular, os gastos estimados considerando diretos e indiretos, nos últimos anos foram de 30 a 37 bilhões de reais ${ }^{5}$.

As DCV usualmente resultam em disfunções vasculares, como por exemplo, alterações na pressão sanguínea, trombose e aterosclerose, essas por sua vez, acabam por comprometer diversas funções orgânicas ${ }^{6}$. Dentre as DCV de maior relevância, destaca-se a aterosclerose, uma patologia inflamatória crônica relacionada com a formação de placas de ateroma, que promovem o espessamento da parede das artérias e redução da elasticidade arterial, levando assim a lesão do endotélio vascular?.

Um dos fatores de risco primordiais no desenvolvimento da doença é a alteração nos níveis dos lipídios séricos (dislipidemia), principalmente aumento da lipoproteína de baixa densidade $(\mathrm{LDL}-\mathrm{C})^{8}$. As lesões iniciais da aterosclerose, podem ocorrer precocemente, inclusive durante a infância, e decorrer de fatores genéticos e ambientais, podendo assim, progredir para complicações como o infarto e Acidente Vascular Cerebral'7.

A dislipidemia é caracterizada por alterações no colesterol total (CT) e frações lipídicas de forma isolada ou mista, como: aumento dos níveis séricos de LDL-C, Triglicerídeos (TG) e/ou diminuição da Lipoproteína de Alta Densidade (HDL-C) ${ }^{9}$. Desta forma, a avaliação do perfil lipídico da população é essencial para a construção de estratégias de saúde, que reduzem os fatores de risco e previnem as DCV.

Tendo em vista que as DCV são umas das principais causas de morte na atualidade e que as alterações do perfil lipídico são, comprovadamente um dos principais fatores de risco relacionados, o presente estudo buscou avaliar o perfil lipídico de indivíduos que realizaram exames laboratoriais em um hospital da cidade de Goiânia, além de classificar os tipos de dislipidemias e estimar a prevalência da mesma entre os diferentes sexos e faixa etária da população estudada.

\section{Métodos}

Este é um estudo observacional do tipo transversal analítico, realizado a partir de dados disponibilizados pelo laboratório que atende a um complexo de saúde para militares de Goiás. Foram selecionados indivíduos que realizaram exames de rotina para o perfil lipídico (CT, LDL-C, HDL-C eTG) no período entre fevereiro de 2017 a março de 2018, independente do gênero e da faixa etária. Foram incluídos neste estudo todos que apresentaram alteração no perfil lipídico (dislipidemia).

As dislipidemias foram classificadas em hipercolesterolemia isolada, hipertrigliceridemia isolada; hiperlipidemia mista e HDL-C baixo. Os indivíduos que apresentaram alterações no perfil lipídico, mas não se encaixaram em um dos grupos citados, entraram para o grupo de alterações não classificáveis. Os valores referenciais do perfil lipídico e a classificação das dislipidemias seguiram os critérios estabelecidos pela Atualização da Diretriz Brasileira de Dislipidemias e Prevenção da Aterosclerose $-2017^{9}$.

Os dados obtidos foram tabulados em Microsoft Excel $2017^{\circledR}$ e analisados no programa estatístico BioStat ${ }^{\oplus}$. Dessa forma, estimou-se a prevalência de dislipidemia nos pacientes, realizando-se testes de normalidade com distribuição normal, seguida de teste de qui-quadrado, para associar e avaliar as diferenças dos níveis lipídicos, com intervalo de confiança de $95 \%$ (IC 95\%) e nível de associação com $p<0,05$. A construção do gráfico foi realizada através do programa GraphPad Prism 6.0.

Este estudo foi submetido e aprovado pelo Comitê de Ética da Pontifícia Universidade Católica de Goiás (Parecer n 235.376).

\section{Resultados}

Foram avaliados um total de 7699 indivíduos, destes 3014 (39\%) eram do sexo feminino e 4685 (61\%) do sexo masculino, como demostrado na tabela 1.

Tabela 1. Distribuição da população do estudo segundo sexo e faixa etária.

\begin{tabular}{cccc}
\hline Faixa etária & Feminino & Masculino & Total \\
\hline $0-19$ anos & 371 & 306 & 677 \\
$20-59$ anos & 2048 & 3933 & 5981 \\
$\geq 60$ anos & 595 & 446 & 1041 \\
Total & 3014 & & 7699 \\
\hline $\mathrm{p}>0,05$ & & 4685 &
\end{tabular}


O número de indivíduos com perfil lipídico alterado foi de 5280 (69\%), esses foram divididos de acordo com sexo e faixa etária, mostrando maior prevalência de dislipidemias no sexo masculino $(73 \%$ no sexo masculino contra $61 \%$ no sexo feminino). Nessa população a faixa etária com maior prevalência de alterações situou-se entre 20 e 59 anos (74\%), enquanto no sexo feminino a maior prevalência está entre os maiores de 60 anos (68\%), como pode ser observado na tabela 2.

Tabela 2. Prevalência de dislipidemias em indivíduos segundo sexo e faixa etária.

\begin{tabular}{ccc}
\hline Faixa etária & Feminino & Masculino \\
\hline $0-19$ anos & $67 \%$ & $71 \%$ \\
$20-59$ anos & $58 \%$ & $74 \%$ \\
$\geq 60$ anos & $68 \%$ & $69 \%$ \\
TOTAL & $61 \%$ & $73 \%$ \\
\hline
\end{tabular}

${ }^{*} p>0,05$

Dos 5280 indivíduos com dislipidemias, 2874 (54,4\%) foram classificados. Dentre as classificações, notou-se maior prevalência de Hiperlipidemia mista na população masculina em relação a feminina, com relevância estatística significante, sendo $p<$ 0,0001 (IC 95\%). Na análise do componente HDL-C baixo, foi constatado que a prevalência foi maior na população feminina quando comparada à masculina, com $p<0,0001$ (IC 95\%). Nos demais grupos, não foi observado associação entre os sexos, como apresentado na tabela 3.

Tabela 3. Classificação das dislipidemias de acordo com o sexo dos indivíduos.

\begin{tabular}{|c|c|c|c|}
\hline Classificação & Sexo $^{a}$ & $\%$ & Valor de $p$ \\
\hline \multirow{2}{*}{ Hipercolesterolemia isolada } & M & $0 \%$ & \\
\hline & $\mathrm{F}$ & $0 \%$ & \\
\hline \multirow{2}{*}{ Hipertrigliceridemia isolada } & M & $10 \%$ & \\
\hline & $\mathrm{F}$ & $10 \%$ & \\
\hline \multirow{2}{*}{ Hiperlipidemia mista } & M & $10 \%$ & \multirow{2}{*}{$\mathrm{p}<0,0001 *$} \\
\hline & $\mathrm{F}$ & $6 \%$ & \\
\hline \multirow[b]{2}{*}{ Hiperlipidemia mista } & M & $32 \%$ & \multirow[b]{2}{*}{$\mathrm{p}<0,0001$ * } \\
\hline & $\mathrm{F}$ & $44 \%$ & \\
\hline
\end{tabular}

a: M: masculino, F: feminino

* sexo masculino apresentou maior prevalência de Hiperlipidemia mista

** sexo feminino apresentou maior prevalência de HDL baixo
Quando comparados os indivíduos com alteração no perfil lipídico com o indivíduos sem nenhuma alteração, não houve diferença significativa entre os sexos e idade. A análise dos dados mostrou uma associação estatisticamente significante entre o envelhecimento e o maior número de classificações das dislipidemias nos indivíduos dos quatro grupos, com $\mathrm{p}<$ 0,0001 (IC 95\%), quando comparados entre às 3 faixas etárias, como demonstrado na figura 1.

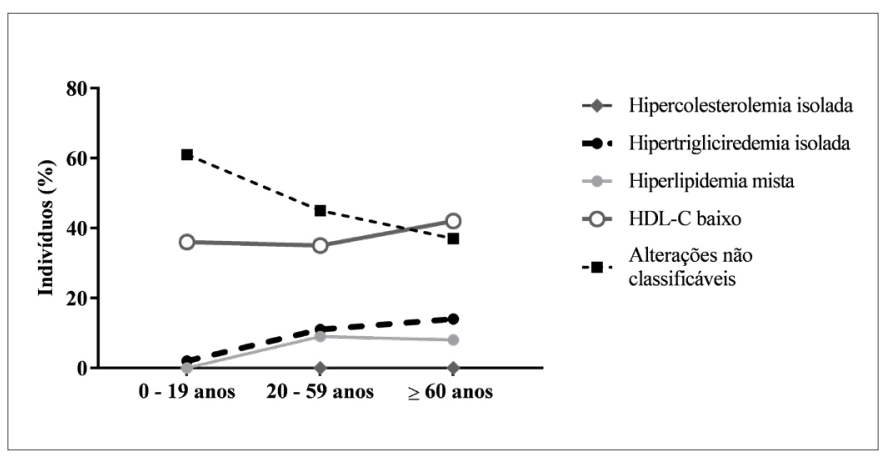

Figura 1. Distribuição das alterações do perfil lipídico dos indivíduos de acordo com a faixa etária. HDL-C: lipoproteína de alta densidade.

\section{Discussão}

As DCV constituem a principal causa de morte entre as doenças crônicas não transmissíveis (DCNT) no Brasil ${ }^{10,}$ mesmo com os avanços no controle e na diminuição do coeficiente de mortalidade ${ }^{11}$. Dessa forma, a avaliação precoce dos fatores de risco modificáveis como a dislipidemia, é importante pois possibilita a prevenção de eventos cardiovasculares e o tratamento adequado dos indivíduos.

O presente estudo demostrou elevada prevalência de dislipidemia na amostra representativa, esses dados são semelhantes com outros trabalhos de base populacionais realizados em Goiânia ${ }^{12}$ e em diferentes cidades como, Ribeirão Preto ${ }^{13}$ e Rio de Janeiro ${ }^{14}$ ao longo dos anos. Demostrando a presença de dislipidemia como grande fator de risco de DCV na população brasileira.

$\mathrm{Na}$ análise da classificação das dislipidemias pode-se observar que o sexo masculino obteve maior prevalência de casos de Hiperlipidemia mista, esses resultados são compatíveis com outros estudos que mostraram alterações principalmente no componente dos TG ${ }^{15-17}$. Isso demostra, alto risco de desenvolvimento de DCV nesses indivíduos, correlacionando com os dados da Pesquisa Nacional de Saúde (PNS) ${ }^{18}$ de 2013, onde $23,1 \%$ dos indivíduos do sexo masculino contra $11,4 \%$ do sexo feminino no estado de Goiás já realizaram alguma cirurgia e procedimentos para o tratamento de doenças coronarianas. 
A Hiperlipidemia mista no sexo masculino, está relacionada ao maior número de fatores de risco associados com os hábitos de vida, como sedentarismo, tabagismo, etilismo e alimentação rica em gorduras ${ }^{19}$. Esses fatores proporcionam o aumento das concentrações de TG (quilomícrons, lipoproteínas de muito baixa densidade e remanescentes) que favorecem a elevada formação de LDL-C, que são mais susceptíveis a oxidação no endotélio vascular ${ }^{7}$. Estudos ${ }^{14,20}$ vem demostrando aumento de TG em adolescentes e adultos jovens, refletindo assim, um aumento populacional do peso corporal nos últimos anos, no presente estudo não observamos resultados semelhantes aos TG em relação a faixa etária.

Neste estudo pode-se observar a maior prevalência de HDLC baixo no sexo feminino, correlacionando com o estudo de Oliveira, et al. ${ }^{16}$, que apresentou $91,38 \%$ de mulheres com HDL-C baixo. As concentrações séricas do HDL-C estão inversamente relacionadas ao risco de doença aterosclerótica, esta lipoproteína atua no transporte reverso do colesterol, como também exerce várias atividades como antioxidantes, anti-inflamatórias, vasodilatadoras entre outras ${ }^{21}$.

As alterações do perfil lipídico na população feminina são muitas vezes relacionadas às suas alterações hormonais, principalmente na pós-menopausa, sendo os baixos níveis HDL-C um dos critérios da síndrome metabólica ${ }^{22}$. Entretanto neste estudo não foram observados correlações dos níveis de HDL-C em relação as faixas etárias. Esses achados são similares a outros estudos que não evidenciaram correlação entre mulheres na pós-menopausa e o surgimento de níveis baixos de HDL-C23,24. $\mathrm{O}$ estudo feito por Fernandez e Murilo ${ }^{25}$ demonstrou, inclusive, que mulheres na pós-menopausa apresentaram níveis mais altos de $\mathrm{HDL}-\mathrm{C}$ em comparação com mulheres na pré-menopausa. Tais estudos tem atribuído esses resultados principalmente ao peso corporal e à gordura visceral, que parecem ter maior influência nas dislipidemias do que propriamente na idade ou no estado hormonal ${ }^{25,26}$.

No presente estudo pode-se observar associação entre o envelhecimento e o maior número de classificações de dislipidemias. Este resultado correlaciona com os dados da $\mathrm{PNS}^{27}$ que demostraram que o avanço da idade, independente do sexo proporciona aumento dos níveis de CT e LDL-C. Devido ao fato dos idosos serem vulneráveis a mais fatores de riscos e doenças, justificando assim o maior número de idosos no Brasil acometidos por DCNT ${ }^{10}$.

\section{Conclusão}

O presente trabalho avaliou a prevalência das dislipidemias nos pacientes atendidos no laboratório de um hospital em Goiânia-GO e constatou que a maioria da população estudada apresentou alterações no perfil lipídico. Foi evidente a maior prevalência de HDL-C baixo no sexo feminino e de Hiperlipidemia mista no sexo masculino, ambos independentemente da idade. Tal observação, demonstra a necessidade de atenção e de investigação precoce das alterações no perfil lipídico, uma vez que grande parte da população possui estas alterações e são consideradas como importantes fatores de risco no desenvolvimento de doenças cardiovasculares e aumento da mortalidade.

\section{Referências}

1. Organização Mundial da Saúde. Doenças cardiovasculares [Internet]. Organização Pan-Americana da Saúde. 2017 [cited 2020 Aug 12]. Available from: https://www.paho.org/bra/index. php?option=com_content\&view=article\&id=5253:do encas-cardiovasculares\&ltemid=1096.

2. Beaglehole R, Bonita R. Global public health: a scorecard. Lancet. 2008;372(9654):1988-96.

3. Butler D. UN targets top killers. Nature. 2011;477(7364):260-1.

4. Barreto IJB, Guarda FRB, Silva PBC, Silva RN, Farias SJM, Silva AEA. Gastos com internações hospitalares por doenças relacionadas à inatividade física no Brasil. Lect Educ Física y Deport. 2020;25(265):29-43.

5. Siqueira ASE, Siqueira-Filho AG, Land MGP. Análise do impacto econômico das doenças cardiovasculares nos últimos cinco anos no Brasil. Arq Bras Cardiol. 2017;109(1):39-46.

6. Michael B. Cardiovascular disease. Nature. 2008;451(7181):903.

7. Libby P, Buring JE, Badimon L, Hansson GK, Deanfield J, Bittencourt MS, et al. Atherosclerosis. Nat Rev Dis Prim. 2019;5(1):1-18.

8. Soppert J, Lehrke M, Marx N, Jankowski J, Noels H. Lipoproteins and lipids in cardiovascular disease: from mechanistic insights to therapeutic targeting. Adv Drug Deliv Rev. 2020;1-30.

9. Faludi AA, Izar MCO, Saraiva JFK, Chacra APM, Bianco HT, Afiune Neto A, et al. Atualização da Diretriz Brasileira de Dislipidemias e Prevenção da Aterosclerose - 2017. Soc Bras Cardiol. 2017;109(2):1-76.

10. Christofoletti M, Duca GF, Gerage AM, Malta DC. Simultaneidade de doenças crônicas não transmissíveis em 2013 nas capitais brasileiras: prevalência e perfil sociodemográfico. Epidemiol e Serv saude Rev do Sist Unico Saude do Bras. 2020;29(1):e2018487.

11. Malta DC, Andrade SSCA, Oliveira TP, Moura L, Prado RR, Souza MFM. Probabilidade de morte prematura por 
doenças crônicas não transmissíveis, Brasil e regiões, projeções para 2025. Rev Bras Epidemiol. 2019;22.

12. Carnelosso ML, Barbosa MA, Porto CC, Silva SA, Carvalho MM, Oliveira ALI. Prevalência de fatores de risco para doenças cardiovasculares na rregião leste de Goiânia (GO). Cien Saude Colet. 2010;15(suppl 1):1073-80.

13. Moraes SA, Checchio MV, Freitas ICM. Dislipidemia e fatores associados em adultos residentes em Ribeirão Preto, SP. Resultados do Projeto EPIDCV. Arq Bras Endocrinol Metabol. 2013;57(9):691-701.

14. Vizentin NP, Cardoso PMS, Maia CAG, Alves IP, Aranha GL, Giannini DT. Dislipidemia em adolescentes atendidos em um hospital universitário no Rio de Janeiro/Brasil: prevalência e associação dyslipidemia. Arq Bras Cardiol. 2019;112(2):147-51.

15. Souza LJ, Souto Filho JTD, Souza TF, Reis AFF, Neto CG, Bastos DA, et al. Prevalência de dislipidemia e fatores de risco em Campos dos Goytacazes - RJ. Arq Bras Cardiol. 2003;81(3):249-64.

16. Oliveira LB, Carvalho IB, Dourado CSME, Dourado JCL, Nascimento MO. Prevalência de dislipidemias e fatores de risco associados. J Heal Biol Sci. 2017;5(4):320.

17. Li J, Wang L, Li Y, Bi Y, Jiang Y, Mi S, et al. Epidemiologic characteristics of dyslipidemia in chinese adults 2010. Zhonghua Yu Fang Yi Xue Za Zhi. 2012;46(5):414-8.

18. Instituto Brasileiro de Geografia e Estatística (IBGE). Percepção do estado de saúde, estilos de vida e doenças crônicas. In: Pesquisa Nacional de Saúde. Rio de Janeiro; 2013. p. 128.

19. Reiner Ž. Hypertriglyceridaemia and risk of coronary artery disease. Nat Rev Cardiol. 2017;14(7):1-11.

20. Gomes ÉlL, ZagoVHS, Faria EC. Avaliação de perfis lipídicos infanto-juvenis solicitados nas unidades básicas de saúde em Campinas/SP, Brasil: um estudo laboratorial transversal. Arq Bras Cardiol. 2020;114(1):47-56.

21. Macedo LET, Faerstein E. Cholesterol and prevention of atherosclerotic events: limits of a new frontier. Rev Saude Publica. 2017;51(1):1-7.

22. Expert Panel on Detection, Evaluation, and Treatment of High Blood Cholesterol in Adults. Executive summary of the third report of the National Cholesterol Education Program (NCEP) expert panel on detection, evaluation, and treatment of high blood cholesterol in adults (adult treatment panel III). JAMA. 2001;285(19):2486-97.

23. Çetin I, Yildirim B, Şahin Ş, Şahin I, Etikan I. Serum lipid and lipoprotein levels, dyslipidemia prevalence, and the factors that influence these parameters in a Turkish population living in the province of Tokat. Turkish J Med
Sci. 2010;40(5):771-82.

24. Derby CA, Crawford SL, Pasternak RC, Sowers M, Sternfeld $B$, Matthews KA. Lipid changes during the menopause transition in relation to age and weight. Am J Epidemiol. 2009;169(11):1352-61.

25. Fernandez ML, Murillo AG. Postmenopausal women have higher $\mathrm{HDL}$ and decreased incidence of low HDL than premenopausal women with metabolic syndrome. Healthcare. 2016;4(20):1-10.

26. Polotsky HN, Polotsky AJ. Metabolic implications of menopause. Semin Reprod Med. 2010;28(5):426-34.

27. Malta DC, Szwarcwald CL, Machado ÍE, Pereira CA, Figueiredo AW, Sá ACMGN, et al. Prevalência de colesterol total e frações alterados na população adulta brasileira: pesquisa nacional de saúde. Rev Bras Epidemiol. 2019;22(Suppl 2):1-13. 